\title{
Approximation of functions in generalized Zygmund class by double Hausdorff matrix
}

\author{
H.K. Nigam ${ }^{1}$, M. Mursaleen ${ }^{2,3,4^{*}}$ (D) and Supriya Rani ${ }^{1}$
}

"Correspondence:

mursaleenm@gmail.com

${ }^{2}$ Department of Medical Research,

China Medical University Hospital,

China Medical University (Taiwan),

Taichung, Taiwan

${ }^{3}$ Department of Mathematics,

Aligarh Muslim University, Aligarh,

India

Full list of author information is

available at the end of the article

\begin{abstract}
In the present work, we emphasize, for the first time, the error estimation of a two-variable function $g(y, z)$ in the generalized Zygmund class $Y_{r}^{(\xi)}(r \geq 1)$ using the double Hausdorff matrix means of its double Fourier series. In fact, in this work, we establish two theorems on error estimation of a two-variable function of $g$ in the generalized Zygmund class.
\end{abstract}

Keywords: Error estimation; Generalized Zygmund class; Double Hausdorff ( $\left.\Delta_{H_{p q}}\right)$ summability means; Double Fourier series (DFS)

\section{Introduction}

The study of the error estimation of a function of single variable $g$ in Lipschitz spaces, Hölder spaces, generalized Hölder spaces, Besov spaces, Zygmund spaces, and generalized Zygmund spaces with different single means, and various product summability means of Fourier series and conjugate Fourier series have been considered as a center of creative study for the researchers [1-13] in the past few decades. The error estimation of a two-variable function $g(y, z)$ in a Hölder space with the double matrix means of the double Fourier series and its generalization for $n$-variable functions in Hölder spaces using multiple Fourier series were obtained in [14], and the degree of approximation of Nòrlund means of double Fourier series continuous two-variable functions was obtained in [15]. The above review of research shows that the studies of error estimation of a twovariable function $g(y, z)$ in the generalized Zygmund class $Y_{r}^{(\xi)}(r \geq 1)$ using double Hausdorff means of double Fourier series have not been initiated so far.

The basic theory of Hausdorff transformations for double sequences came into being by Adams [16] in 1933. Later, a few authors investigated double Hausdorff matrices; see, for example, Ramanujan [17] and Ustina [18]. Consequently, we consider the error estimation of two-variable functions $g(y, z)$ in the generalized Zygmund class $Y_{r}^{(\xi)}(r \geq 1)$ by the double Hausdorff summability means of its double Fourier series.

We establish two theorems on the degree of approximation of a two-variable function in the generalized Zygmund class $Y_{r}^{(\xi)}(r \geq 1)$ by the double Hausdorff summability means of its double Fourier series.

(c) The Author(s) 2020. This article is licensed under a Creative Commons Attribution 4.0 International License, which permits use sharing, adaptation, distribution and reproduction in any medium or format, as long as you give appropriate credit to the original author(s) and the source, provide a link to the Creative Commons licence, and indicate if changes were made. The images or other third party material in this article are included in the article's Creative Commons licence, unless indicated otherwise in a credit line to the material. If material is not included in the article's Creative Commons licence and your intended use is not permitted by statutory regulation or exceeds the permitted use, you will need to obtain permission directly from the copyright holder. To view a copy of this licence, visit http://creativecommons.org/licenses/by/4.0/. 
Let $z: \mathbb{N} \times \mathbb{N} \mapsto \mathbb{C}$ be a double sequence of complex numbers, and let $\left(s_{p, q}\right)$ be the double sequence defined by

$$
s_{p, q}:=\sum_{i=1}^{p}\left(\sum_{j=1}^{q} z_{i, j}\right)
$$

The pair $(z, s)$ is called a double series and is denoted by the symbol $\sum_{p, q=1}^{\infty} z_{p, q}$.

Let $\sum_{p=1}^{\infty} \sum_{q=1}^{\infty} z_{p, q}$ be an infinite double series having the $(p, q)$ th partial sum $s_{p, q}=$ $\sum_{i=1}^{p}\left(\sum_{j=1}^{q} z_{i, j}\right)$.

Let $g(y)$ be a $2 \pi$-periodic Lebesgue-integrable function of $y$ over the interval $(-\pi, \pi)$. The Fourier series of $g(y)$ is given by

$$
g(y) \sim \frac{a_{0}}{2}+\sum_{q=1}^{\infty}\left(a_{q} \cos q y+b_{q} \sin q y\right)
$$

and the conjugate series to (1) is given by

$$
\sum_{q=1}^{\infty}\left(a_{q} \cos q y-b_{q} \sin q y\right)
$$

It is well known that corresponding conjugate function of (2) is defined as

$$
\tilde{g}(y)=\frac{1}{\pi} \int_{0}^{\pi} \frac{g(y+l)-g(y-l)}{2 \tan \frac{l}{2}} d l .
$$

Let $g(y, z)$ be a function of $(y, z)$, periodic with respect to $y$ and with respect to $z$, in each case with period $2 \pi$, integrable in the Lebesgue sense and summable in the square $Q(-\pi,-\pi ; \pi, \pi)$.

The double Fourier series of a function $g(y, z)$ is given by

$$
\begin{aligned}
g(y, z) \sim & \sum_{p=0}^{\infty} \sum_{q=0}^{\infty} \beta_{p, q}\left[\tau_{p, q} \cos p y \cos q z+\psi_{p, q} \sin p y \cos q z\right. \\
& \left.+\rho_{p, q} \cos p y \sin q z+\zeta_{p, q} \sin p y \sin q z\right] \\
= & \sum_{p=0}^{\infty} \sum_{q=0}^{\infty} \tau_{p, q} A_{p, q}(y, z),
\end{aligned}
$$

where

$$
\beta_{p, q}= \begin{cases}\frac{1}{4} & \text { for } p=0, q=0 \text { or } p=q=0 \\ \frac{1}{2} & \text { for } p>0, q=0 \text { and } p=0, q>0 \\ 1 & \text { for } p>0, q>0\end{cases}
$$


and the coefficients $\tau_{p, q}, \psi_{p, q}, \rho_{p, q}$, and $\zeta_{p, q}$ are calculated by the formulae

$$
\begin{aligned}
& \tau_{p, q}=\frac{1}{\pi^{2}} \iint_{Q} g(y, z) \cos p y \cos q z d y d z, \\
& \psi_{p, q}=\frac{1}{\pi^{2}} \iint_{Q} g(y, z) \sin p y \cos q z d y d z, \\
& \rho_{p, q}=\frac{1}{\pi^{2}} \iint_{Q} g(y, z) \cos p y \sin q z d y d z, \\
& \zeta_{p, q}=\frac{1}{\pi^{2}} \iint_{Q} g(y, z) \sin p y \sin q z d y d z
\end{aligned}
$$

for $p=0,1,2, \ldots$ and $q=0,1,2, \ldots$. The quantities

$$
\begin{aligned}
s_{p, q}(y, z)= & \sum_{j=0}^{p} \sum_{k=0}^{q}\left[\tau_{j, k} \cos j y \cos k z+\psi_{j, k} \sin j y \cos k z\right. \\
& \left.+\rho_{j, k} \cos j y \sin k z+\zeta_{j, k} \sin j y \sin k z\right]
\end{aligned}
$$

$(p=0,1,2, \ldots ; q=0,1,2, \ldots)$ are called the partial sums of the double Fourier series.

According to (5), we know that

$$
s_{p, q}(y, z)-g(y, z)=\frac{1}{\pi^{2}} \iint_{Q} g(y+s, z+l) \frac{\left[\sin \left(p+\frac{1}{2}\right) s\right]\left[\sin \left(q+\frac{1}{2}\right) l\right]}{4 \sin \frac{s}{2} \sin \frac{l}{2}} d s d l .
$$

The double Hausdorff matrix has the entries

$$
h_{p q i j}=\left(\begin{array}{c}
p \\
i
\end{array}\right)\left(\begin{array}{l}
q \\
j
\end{array}\right) \Delta_{1}^{p-i} \Delta_{2}^{q-j} \mu_{i j}
$$

where $\left\{\mu_{i j}\right\}$ is any real or complex sequence, and for any sequence $\mu_{i j}$, the operator $\Delta$ is defined by

$$
\Delta_{i j} \mu_{i j}:=\mu_{i j}-\mu_{i+1, j}-\mu_{i, j+1}+\mu_{i+1, j+1}
$$

and

$$
\Delta_{1}^{p-i} \Delta_{2}^{q-j} \mu_{i j}=\sum_{s=0}^{p-1} \sum_{l=0}^{q-j}(-1)^{i+j}\left(\begin{array}{c}
p-i \\
s
\end{array}\right)\left(\begin{array}{c}
q-j \\
l
\end{array}\right) \mu_{i+s, j+l}
$$

Necessary and sufficient condition for double Hausdorff matrices to be conservative is the existence of a function $\chi(s, l) \in B V[0,1] \times[0,1]$ such that

$$
\int_{0}^{1} \int_{0}^{1}|d \chi(s, l)|<\infty
$$

and

$$
\mu_{p q}=\int_{0}^{1} \int_{0}^{1} s^{p} l^{q} d \chi(s, l)
$$


Without loss of generality, we may assume that $\chi(0,0)=0$. If, in addition, we have $\chi(1,1)=$ 1 , and the continuity conditions

$$
\begin{aligned}
& \chi(s,+0)=\chi(s, 0), \quad \chi(s,+0)=\lim _{l \rightarrow 0} \chi(s, l), \\
& \chi(+0, l)=\chi(0, l), \quad \chi(+0, l)=\lim _{s \rightarrow 0} \chi(s, l)
\end{aligned}
$$

are also satisfied, so that $\mu_{00}=1$.

We say that $\mu_{p q}$ is a regular moment constant $[16,17]$.

Let $\sum_{p=0}^{\infty} \sum_{q=0}^{\infty} b_{p, q}$ be a double series with $s_{p, q}=\sum_{j=0}^{p} \sum_{k=0}^{q} b_{j, k}$ as its $(p, q)$ th partial sums.

The double Hausdorff mean $t_{p, q}$ is given by

$$
t_{p, q}=\sum_{j=0}^{p} \sum_{k=0}^{q} h_{p, q, j, k} s_{j, k}
$$

The double series $\sum_{p=0}^{\infty} \sum_{q=0}^{\infty} b_{p, q}$ with the sequence of $(p, q)$ th partial sums $\left(s_{p, q}\right)$ is said to be summable by the double Hausdorff summability method or summable $\left(H_{p, q}\right)$ if $t_{p, q}$ tends to a limit $s$ as $p \rightarrow \infty$ and $q \rightarrow \infty$.

The norm $\|\cdot\|_{r}$ is defined as

$$
\|f\|_{r}:= \begin{cases}\left\{\frac{1}{4 \pi^{2}} \int_{0}^{2 \pi} \int_{0}^{2 \pi}|g(y, z)|^{r} d y d z\right\}^{1 / r} & \text { for } 1 \leq r<\infty \\ {\operatorname{ess} \sup _{0<y<2 \pi}|g(y, z)|}_{0<z<2 \pi} & \text { for } r=\infty\end{cases}
$$

Let $\xi:[-\pi,-\pi ; \pi, \pi] \rightarrow \mathbb{R} \times \mathbb{R}$ be an arbitrary function with $\xi(s, l)>0$ for $0<s<2 \pi$, $0<l<2 \pi$ and such that $\lim _{\substack{s \rightarrow 0^{+} \\ l \rightarrow 0^{+}}} \xi(s, l)=\xi(0,0)=0$.

We define

$$
\begin{aligned}
Y_{r}^{(\xi)}:= & \left\{g \in L^{r}: \sup _{\substack{s \neq 0 \\
l \neq 0}} \frac{\|g(\cdot+s, \cdot+l)+g(\cdot+s, \cdot-l)+g(\cdot-s, \cdot+l)+g(\cdot-s, \cdot-l)+4 g(\cdot, \cdot)\|_{r}}{\xi(s, l)}\right. \\
& <\infty\}
\end{aligned}
$$

and

$$
\|g\|_{r}^{(\xi)}:=\|g\|_{r}+\sup _{\substack{s \neq 0 \\ l \neq 0}} \frac{\|g(\cdot+s, \cdot+l)+g(\cdot+s, \cdot-l)+g(\cdot-s, \cdot+l)+g(\cdot-s, \cdot-l)+4 g(\cdot, \cdot)\|_{r}}{\xi(s, l)} .
$$

Clearly, $\|\cdot\|_{r}^{(\xi)}$ is a norm on $Y_{r}^{(\xi)}$.

Hence the Zygmund space $\left(Y_{r}^{(\xi)}\right)$ is a Banach space under the norm $\|\cdot\|_{r}^{(\xi)}$. The completeness of the space $Y_{r}^{(\xi)}$ can be discussed considering the completeness of $L^{r}(r \geq 1)$. We refer to [19] for more detail on the Zygmund space.

We write

$$
\begin{aligned}
\phi(s, l) & =\phi(y, z: s, l) \\
& =\frac{1}{4}[g(y+s, z+l)+g(y+s, z-l)+g(y-s, z+l)+g(y-s, z-l)-4 g(y, z)]
\end{aligned}
$$




$$
\begin{aligned}
& \Phi(y, z)=\int_{0}^{y} \int_{0}^{z}|\phi(v, w)| d v d w ; \\
& K_{p, q}(s, l)=\frac{1}{4 \pi^{2}} \sum_{j=0}^{p} h_{p, j} \frac{\sin \left(j+\frac{1}{2}\right) s}{\sin \frac{s}{2}} \sum_{k=0}^{q} h_{q, k} \frac{\sin \left(k+\frac{1}{2}\right) l}{\sin \frac{l}{2}} .
\end{aligned}
$$

Remark 1.1 A double Hausdorff matrix method reduces to

(i) $(\mathrm{C}, 1,1)$ summability mean if $\sigma_{p}=\frac{1}{p+1}$ and $\sigma_{q}=\frac{1}{q+1}$ and

(ii) (E,r,r) summability mean if $E_{p}^{r}=\frac{1}{(1+r)^{p}}\left(\begin{array}{l}p \\ j\end{array}\right) r^{p-j}$ and $E_{q}^{r}=\frac{1}{(1+r)^{q}}\left(\begin{array}{l}q \\ k\end{array}\right) r^{q-k}$.

\section{Theorems}

Theorem 2.1 Let $g$ be a function of $(y, z)$ periodic (in each case, with period $2 \pi$ ) with respect to $y$ and $z$, Lebesgue integrable on $Q$, and belonging to the class $Y_{r}^{(\xi)}, r \geq 1$. Then the error estimate of $g$ by the $\Delta_{H_{p, q}}$ method of its DFS is given by

$$
\left\|t_{p, q}^{\Delta H_{p, q}}(y, z)-g(y, z)\right\|_{r}^{(\eta)}=O\left(\frac{p+q+6}{(p+1)(q+1)} \int_{\frac{1}{p+1}}^{\pi} \int_{\frac{1}{q+1}}^{\pi} \frac{\xi(s, l)}{\eta(s, l) s^{2} l^{2}}\right)
$$

where $\xi$ and $\eta$ denote the moduli of continuity of second order such that $\xi(s, l) / \eta(s, l)$ is positive and nondecreasing.

Theorem 2.2 In addition to the conditions of Theorem 2.1, if $\xi(s, l) / s \ln (s, l)$ is nonincreasing, then the error estimate of $g$ in $Y_{r}^{(\xi)}(r \geq 1)$ by the $\Delta_{H_{p, q}}$ method of its DFS is given by

$$
\left\|t_{p, q}^{\Delta H_{p, q}}(y, z)-g(y, z)\right\|_{r}^{(\eta)}=O\left(\frac{\xi\left(\frac{1}{p+1}, \frac{1}{q+1}\right)}{\eta\left(\frac{1}{p+1}, \frac{1}{q+1}\right)}(p+q+6) \log \pi(p+q+2)\right) .
$$

\section{Lemmas}

Lemma 3.1 $K_{p q}(s, l)=O((p+1)(q+1))$ for $0<s \leq \frac{1}{p+1}$ and $0<l \leq \frac{1}{q+1}$.

Proof For $0<s \leq \frac{1}{p+1}, 0<l \leq \frac{1}{l+1}, \sin \frac{l}{2} \geq \frac{l}{\pi}$, and $\sin q l \leq q l$, we get

$$
\begin{aligned}
\left|K_{p q}(s, l)\right|= & \frac{1}{4 \pi^{2}}\left|\sum_{j=0}^{p} h_{p, j} \frac{\sin \left(j+\frac{1}{2}\right) s}{\sin \frac{s}{2}} \sum_{k=0}^{q} h_{q, k} \frac{\sin \left(k+\frac{1}{2}\right) l}{\sin \frac{l}{2}}\right| \\
= & \frac{1}{4 \pi^{2}} \mid \sum_{j=0}^{p} \int_{0}^{1}\left(\begin{array}{l}
p \\
j
\end{array}\right) v^{j}(1-v)^{p-j} d \chi(v) \frac{\sin \left(j+\frac{1}{2}\right) s}{\sin \frac{s}{2}} \\
& \times \sum_{k=0}^{q} \int_{0}^{1}\left(\begin{array}{l}
q \\
k
\end{array}\right) w^{k}(1-w)^{q-k} d \chi(w) \frac{\sin \left(k+\frac{1}{2}\right) l}{\sin \frac{l}{2}} \mid \\
= & \frac{1}{4 \pi^{2}} \mid \sum_{j=0}^{p} \int_{0}^{1}\left(\begin{array}{l}
p \\
j
\end{array}\right) v^{j}(1-v)^{p-j} d \chi(v) \frac{(2 s+1) \frac{s}{2}}{\frac{s}{\pi}} \\
& \times \sum_{k=0}^{q} \int_{0}^{1}\left(\begin{array}{l}
q \\
k
\end{array}\right) w^{k}(1-w)^{q-k} d \chi(w) \frac{(2 k+1) \frac{l}{2}}{\frac{l}{\pi}} \mid
\end{aligned}
$$




$$
\begin{aligned}
= & \frac{1}{16} \mid \sum_{j=0}^{p} \int_{0}^{1}\left(\begin{array}{l}
p \\
j
\end{array}\right) v^{j}(1-v)^{p-j} d \chi(v)(2 s+1) \\
& \times \sum_{k=0}^{q} \int_{0}^{1}\left(\begin{array}{l}
q \\
k
\end{array}\right) w^{k}(1-w)^{q-k} d \chi(w)(2 k+1) \mid .
\end{aligned}
$$

Since

$$
\begin{aligned}
& \sum_{j=0}^{p}\left(\begin{array}{l}
p \\
j
\end{array}\right) v^{j}(1-v)^{p-j}(2 s+1)=(2 p v+1) \quad \text { and } \\
& \sum_{k=0}^{q}\left(\begin{array}{l}
q \\
k
\end{array}\right) w^{k}(1-w)^{q-k}(2 k+1)=(2 q w+1),
\end{aligned}
$$

from (7) and (8) we get

$$
\begin{aligned}
\left|K_{p q}(s, l)\right| & =\frac{1}{16}\left|\int_{0}^{1}(2 p v+1) d v \int_{0}^{1}(2 q w+1) d w\right| \\
& =O((p+1)(q+1)) .
\end{aligned}
$$

Lemma 3.2 $K_{p q}(s, l)=O\left((q+1) \frac{1}{(p+1) s^{2}}\right)$ for $\frac{1}{p+1}<s \leq \pi$ and $0<l \leq \frac{1}{q+1}$.

Proof Since $\frac{1}{p+1}<s \leq \pi, \sin \frac{s}{2} \geq \frac{s}{\pi}, \sin ^{2} q s \leq 1, \sup _{0 \leq v \leq 1}\left|j^{\prime}(v)\right|=M, 0<l \leq \frac{1}{q+1}, \sin \frac{l}{2} \geq \frac{l}{\pi}$, and $\sin q l \leq q l$, we get

$$
\begin{aligned}
\left|K_{p q}(s, l)\right|= & \frac{1}{4 \pi^{2}} \mid \sum_{j=0}^{p} \int_{0}^{1}\left(\begin{array}{l}
p \\
j
\end{array}\right) v^{j}(1-v)^{p-j} d \chi(v) \frac{\sin \left(j+\frac{1}{2}\right) s}{\sin \frac{s}{2}} \\
& \times \sum_{k=0}^{q} \int_{0}^{1}\left(\begin{array}{l}
q \\
k
\end{array}\right) w^{k}(1-w)^{q-k} d \chi(w) \frac{\sin \left(k+\frac{1}{2}\right) l}{\sin \frac{l}{2}} \mid \\
= & \frac{1}{4 \pi^{2}} \mid \sum_{j=0}^{p} \int_{0}^{1}\left(\begin{array}{l}
p \\
j
\end{array}\right) v^{j}(1-v)^{p-j} d \chi(v) \frac{\sin \left(j+\frac{1}{2}\right) s}{\frac{s}{\pi}} \\
& \times \sum_{k=0}^{q} \int_{0}^{1}\left(\begin{array}{l}
q \\
k
\end{array}\right) w^{k}(1-w)^{q-k} d \chi(w) \frac{\left(k+\frac{1}{2}\right) l}{\frac{l}{\pi}} \mid \\
= & \frac{1}{8 s} \mid \sum_{j=0}^{p} \int_{0}^{1}\left(\begin{array}{l}
p \\
j
\end{array}\right) v^{j}(1-v)^{p-j} d \chi(v) \sin \left(j+\frac{1}{2}\right) s \\
& \times \sum_{k=0}^{q} \int_{0}^{1}\left(\begin{array}{l}
q \\
k
\end{array}\right) w^{k}(1-w)^{q-k} d \chi(w)(2 k+1) \mid \\
\leq & \frac{M}{8 s}\left|\sum_{j=0}^{p} \int_{0}^{1}\left(\begin{array}{l}
p \\
j
\end{array}\right) v^{j}(1-v)^{p-j} e^{i\left(j+\frac{1}{2}\right) s} d v \int_{0}^{1}(2 q w+1) d w\right| \\
& \operatorname{since} \sum_{k=0}^{q}\left(\begin{array}{l}
q \\
k
\end{array}\right) w^{k}(1-w)^{q-k}(2 k+1)=(2 q w+1) \\
& \\
&
\end{aligned}
$$




$$
\begin{aligned}
& \leq \frac{M}{8 s}\left|\sum_{j=0}^{p} \int_{0}^{1}\left(\begin{array}{l}
p \\
j
\end{array}\right) v^{j}(1-v)^{p-j} e^{i\left(j+\frac{1}{2}\right) s} d v(q+1)\right| \\
& \leq \frac{M O(q+1)}{8 s}\left|\sum_{j=0}^{p} \int_{0}^{1}\left(\begin{array}{c}
p \\
j
\end{array}\right) v^{j}(1-v)^{p-j} e^{i\left(j+\frac{1}{2}\right) s} d v\right| .
\end{aligned}
$$

Now

$$
\begin{aligned}
& \sum_{j=0}^{p} \int_{0}^{1}\left(\begin{array}{l}
p \\
j
\end{array}\right) v^{j}(1-v)^{p-j} e^{\left(j+\frac{1}{2}\right) s} d v \\
& =(1-v)^{p} \int_{0}^{1} \sum_{j=0}^{p}\left(\begin{array}{c}
p \\
j
\end{array}\right)\left(\frac{v}{1-v}\right)^{j} \operatorname{Im}\left\{e^{i\left(j+\frac{1}{2}\right) s}\right\} d v \\
& =(1-v)^{p} \int_{0}^{1} \sum_{j=0}^{p}\left(\begin{array}{l}
p \\
j
\end{array}\right)\left(\frac{v}{1-v}\right)^{j} \operatorname{Im}\left\{e^{i j s} \cdot e^{\frac{i s}{2}}\right\} d v \\
& =(1-v)^{p} e^{\frac{i s}{2}} \int_{0}^{1} \sum_{j=0}^{p}\left(\begin{array}{c}
p \\
j
\end{array}\right)\left(\frac{v e^{i s}}{1-v}\right)^{j} d v \\
& =(1-v)^{p} \operatorname{Im} \int_{0}^{1}\left[e^{\frac{i s}{2}}\left\{\left(\begin{array}{l}
p \\
0
\end{array}\right)+\left(\begin{array}{l}
p \\
1
\end{array}\right) \frac{v e^{i s}}{1-v}+\left(\begin{array}{l}
p \\
2
\end{array}\right)\left(\frac{v e^{i s}}{1-v}\right)^{2}+\cdots+\left(\begin{array}{l}
p \\
p
\end{array}\right)\left(\frac{v e^{i s}}{1-v}\right)^{p}\right\} d v\right] \\
& =\operatorname{Im}\left[e^{\frac{i s}{2}} \int_{0}^{1}\left\{\left(\begin{array}{l}
p \\
0
\end{array}\right)(1-v)^{p-0}+\left(\begin{array}{c}
p \\
1
\end{array}\right) v e^{i s}(1-v)^{p-1}+\cdots+\left(\begin{array}{c}
p \\
p
\end{array}\right)\left(v e^{i s}\right)^{p}(1-v)^{p-p}\right\} d v\right] \\
& =\operatorname{Im}\left[e^{\frac{i s}{2}} \int_{0}^{1}\left(1-v+v e^{i s}\right)^{p} d v\right] \\
& =\operatorname{Im}\left[e^{\frac{i s}{2}} \int_{0}^{1}\left\{1+v\left(e^{i s}-1\right)\right\}^{p} d v\right] \\
& =\operatorname{Im}\left[\frac{e^{i(p+1) s}-1}{(1+p)\left(e^{\frac{i s}{2}}-e^{\frac{-i s}{2}}\right)}\right] \\
& =\operatorname{Im}\left[\frac{e^{i(p+1) s}-1}{(p+1) 2 i \sin \frac{s}{2}}\right] \\
& =\operatorname{Im}\left[\frac{\cos (p+1) s+i \sin (p+1) s-1}{2 i(p+1) \sin \frac{s}{2}}\right] \\
& =\frac{\sin ^{2}(p+1) \frac{s}{2}}{(p+1) \sin \frac{s}{2}}
\end{aligned}
$$

Now, from equations (9) and (10), we get

$$
\begin{aligned}
\left|K_{p q}(s, l)\right| & \leq \frac{M O(q+1)}{8 s}\left|\frac{\sin ^{2}(p+1) \frac{s}{2}}{(p+1) \frac{s}{2}}\right| \\
& =\frac{M O(q+1)}{8 s}\left|\frac{1}{(p+1) \frac{s}{2}}\right| \\
& =O\left((q+1) \frac{1}{(p+1) s^{2}}\right) .
\end{aligned}
$$

Lemma 3.3 $K_{p q}(s, l)=O\left((p+1) \frac{1}{(q+1) l^{2}}\right)$ for $0<s \leq \frac{1}{p+1}$ and $\frac{1}{q+1}<l \leq \pi$. 
Proof Since $0<s \leq \frac{1}{p+1}$, sin $\frac{s}{2} \geq \frac{s}{\pi}$, sin $p s \leq p s, \frac{1}{q+1}<l \leq \pi, \sin \frac{l}{2} \geq \frac{l}{\pi}, \sin ^{2} q l \leq 1$, and $\sup _{0 \leq w \leq 1}\left|k^{\prime}(w)\right|=N$, we get

$$
\begin{aligned}
& \left|K_{p q}(s, l)\right|=\frac{1}{4 \pi^{2}} \mid \sum_{j=0}^{p} \int_{0}^{1}\left(\begin{array}{l}
p \\
j
\end{array}\right) v^{j}(1-v)^{p-j} d \chi(v) \frac{\sin \left(j+\frac{1}{2}\right) s}{\sin \frac{s}{2}} \\
& \times \sum_{k=0}^{q} \int_{0}^{1}\left(\begin{array}{l}
q \\
k
\end{array}\right) w^{k}(1-w)^{l-k} d \chi(w) \frac{\sin \left(k+\frac{1}{2}\right) l}{\sin \frac{l}{2}} \\
& =\frac{1}{4 \pi^{2}} \mid \sum_{j=0}^{p} \int_{0}^{1}\left(\begin{array}{l}
p \\
j
\end{array}\right) v^{j}(1-v)^{p-j} d \chi(v) \frac{\left(j+\frac{1}{2}\right) s}{\frac{s}{\pi}} \\
& \times \sum_{k=0}^{q} \int_{0}^{1}\left(\begin{array}{l}
q \\
k
\end{array}\right) w^{k}(1-w)^{q-k} d \chi(w) \frac{\sin \left(k+\frac{1}{2}\right) l}{\frac{l}{\pi}} \\
& =\frac{1}{8 l} \mid \sum_{j=0}^{p} \int_{0}^{1}\left(\begin{array}{l}
p \\
j
\end{array}\right) v^{j}(1-v)^{p-j} d \chi(v)(2 j+1) \\
& \times \sum_{k=0}^{q} \int_{0}^{1}\left(\begin{array}{l}
q \\
k
\end{array}\right) w^{k}(1-w)^{q-k} d \chi(w) \sin \left(k+\frac{1}{2}\right) l \\
& \leq \frac{N}{8 l}\left|\int_{0}^{1}(2 p v+1) d v \sum_{k=0}^{q}\left(\begin{array}{l}
q \\
k
\end{array}\right) w^{k}(1-w)^{q-k} e^{i\left(k+\frac{1}{2}\right) l} d w\right| \\
& \text { since } \sum_{j=0}^{p}\left(\begin{array}{l}
p \\
j
\end{array}\right) v^{j}(1-v)^{p-j}(2 s+1)=(2 p v+1) \\
& \leq \frac{N}{8 l}\left|(p+1) \sum_{k=0}^{q}\left(\begin{array}{l}
q \\
k
\end{array}\right) w^{k}(1-w)^{q-k} e^{i\left(k+\frac{1}{2}\right) l} d w\right| \\
& \leq \frac{N O(p+1)}{8 l}\left|\sum_{k=0}^{q} \int_{0}^{1}\left(\begin{array}{l}
q \\
k
\end{array}\right) w^{k}(1-w)^{q-k} e^{i\left(k+\frac{1}{2}\right) l} d w\right| .
\end{aligned}
$$

Now

$$
\begin{aligned}
\sum_{k=0}^{q} & \int_{0}^{1}\left(\begin{array}{l}
q \\
k
\end{array}\right) w^{k}(1-w)^{q-k} e^{\left(k+\frac{1}{2}\right) l} d w \\
= & (1-w)^{q} \int_{0}^{1} \sum_{k=0}^{q}\left(\begin{array}{l}
q \\
k
\end{array}\right)\left(\frac{w}{1-w}\right)^{k} \operatorname{Im}\left\{e^{i\left(k+\frac{1}{2}\right) l}\right\} d w \\
= & (1-w)^{q} \int_{0}^{1} \sum_{k=0}^{q}\left(\begin{array}{l}
q \\
k
\end{array}\right)\left(\frac{w}{1-w}\right)^{k} \operatorname{Im}\left\{e^{i k l} \cdot e^{i \frac{i l}{2}}\right\} d w \\
= & (1-w)^{q} e^{\frac{i l}{2}} \int_{0}^{1} \sum_{k=0}^{q}\left(\begin{array}{l}
q \\
k
\end{array}\right)\left(\frac{w e^{i l}}{1-w}\right)^{k} d w \\
= & (1-w)^{q} \operatorname{Im} \int_{0}^{1}\left[e ^ { i l } \left\{\left(\begin{array}{l}
q \\
0
\end{array}\right)+\left(\begin{array}{l}
q \\
1
\end{array}\right) \frac{w e^{i l}}{1-w}+\left(\begin{array}{l}
q \\
2
\end{array}\right)\left(\frac{w e^{i l}}{1-w}\right)^{2}+\cdots\right.\right. \\
& \left.\left.+\left(\begin{array}{l}
q \\
q
\end{array}\right)\left(\frac{w e^{i l}}{1-w}\right)^{q}\right\} d w\right]
\end{aligned}
$$




$$
\begin{aligned}
= & \operatorname{Im}\left[e ^ { \frac { i l } { 2 } } \int _ { 0 } ^ { 1 } \left\{\left(\begin{array}{l}
q \\
0
\end{array}\right)(1-w)^{q-0}+\left(\begin{array}{l}
q \\
1
\end{array}\right) w e^{i l}(1-w)^{q-1}+\cdots\right.\right. \\
& \left.\left.+\left(\begin{array}{l}
q \\
q
\end{array}\right)\left(w e^{i l}\right)^{q}(1-w)^{q-q}\right\} d w\right] \\
= & \operatorname{Im}\left[e^{\frac{i l}{2}} \int_{0}^{1}\left(1-w+w e^{i l}\right)^{q} d w\right] \\
= & \operatorname{Im}\left[e^{\frac{i l}{2}} \int_{0}^{1}\left\{1+w\left(e^{i l}-1\right)\right\}^{q} d w\right] \\
= & \operatorname{Im}\left[\frac{e^{i(q+1) l}-1}{(1+q)\left(e^{\frac{i l}{2}}-e^{\frac{-i l}{2}}\right)}\right] \\
= & \operatorname{Im}\left[\frac{e^{i(q+1) l}-1}{(q+1) 2 i \sin \frac{l}{2}}\right] \\
= & \operatorname{Im}\left[\frac{\cos (q+1) l+i \sin (q+1) l-1}{2 i(q+1) \sin \frac{l}{2}}\right] \\
= & \frac{\sin ^{2}(q+1) \frac{l}{2}}{(q+1) \sin \frac{l}{2}} .
\end{aligned}
$$

Now from equations (11) and (12) we get

$$
\begin{aligned}
\left|K_{p q}(s, l)\right| & \leq \frac{N O(p+1)}{8 l}\left|\frac{\sin ^{2}(q+1) \frac{l}{2}}{(q+1) \frac{l}{2}}\right| \\
& =\frac{N O(p+1)}{8 l}\left|\frac{1}{(q+1) \frac{l}{2}}\right| \\
& =O\left((p+1) \frac{1}{(q+1) l^{2}}\right) .
\end{aligned}
$$

Lemma 3.4 $K_{p q}(s, l)=O\left(\frac{1}{(p+1) s^{2}} \frac{1}{(q+1) l^{2}}\right)$ for $\frac{1}{p+1}<s \leq \pi$ and $\frac{1}{q+1}<l \leq \pi$.

Proof Since $\frac{1}{p+1}<s \leq \pi, \sin \frac{s}{2} \geq \frac{s}{\pi}, \sin ^{2} p s \leq 1, \sup _{0 \leq v \leq 1}\left|j^{\prime}(v)\right|=M, \frac{1}{q+1}<l \leq \pi, \sin \frac{l}{2} \geq \frac{l}{\pi}$, $\sin ^{2} q l \leq 1$, and $\sup _{0 \leq w \leq 1}\left|k^{\prime}(w)\right|=N$, using (10) and (12), we get

$$
\begin{aligned}
\left|K_{p q}(s, l)\right|= & \frac{1}{4 \pi^{2}} \mid \sum_{j=0}^{p} \int_{0}^{1}\left(\begin{array}{l}
p \\
j
\end{array}\right) v^{j}(1-v)^{p-j} d \chi(v) \frac{\sin \left(j+\frac{1}{2}\right) s}{\sin \frac{s}{2}} \\
& \times \sum_{k=0}^{q} \int_{0}^{1}\left(\begin{array}{l}
q \\
k
\end{array}\right) w^{k}(1-w)^{q-k} d \chi(w) \frac{\sin \left(k+\frac{1}{2}\right) l}{\sin \frac{l}{2}} \mid \\
= & \frac{1}{4 \pi^{2}} \mid \sum_{j=0}^{p} \int_{0}^{1}\left(\begin{array}{l}
p \\
j
\end{array}\right) v^{j}(1-v)^{p-j} d \chi(v) \frac{\left(j+\frac{1}{2}\right) s}{\frac{s}{\pi}} \\
& \times \sum_{k=0}^{q} \int_{0}^{1}\left(\begin{array}{l}
q \\
k
\end{array}\right) w^{k}(1-w)^{q-k} d \chi(w) \frac{\left(k+\frac{1}{2}\right) l}{\frac{l}{\pi}} \mid \\
= & \frac{1}{16 s l} \mid \sum_{j=0}^{p} \int_{0}^{1}\left(\begin{array}{l}
p \\
j
\end{array}\right) v^{j}(1-v)^{p-j} d \chi(v)\left(j+\frac{1}{2}\right) s
\end{aligned}
$$




$$
\begin{aligned}
& \times \sum_{k=0}^{q} \int_{0}^{1}\left(\begin{array}{l}
q \\
k
\end{array}\right) w^{k}(1-w)^{q-k} d \chi(w)(2 k+1) \mid \\
\leq & \frac{M N}{16 s l}\left|\sum_{j=0}^{p} \int_{0}^{1}\left(\begin{array}{l}
p \\
j
\end{array}\right) v^{j}(1-v)^{p-j} e^{i\left(j+\frac{1}{2}\right) s} d v \sum_{k=0}^{q} \int_{0}^{1}\left(\begin{array}{l}
q \\
k
\end{array}\right) w^{k}(1-w)^{q-k} e^{i\left(k+\frac{1}{2}\right) l} d w\right| \\
\leq & \frac{M N}{16 s l}\left|\frac{\sin ^{2}(p+1) \frac{s}{2}}{(p+1) \frac{s}{2}} \frac{\sin ^{2}(q+1) \frac{l}{2}}{(q+1) \frac{l}{2}}\right| \\
= & \frac{M N}{16 s l}\left|\frac{1}{(p+1) \frac{s}{2}} \frac{1}{(q+1) \frac{l}{2}}\right| \\
= & O\left(\frac{1}{(p+1) s^{2}} \frac{1}{(q+1) l^{2}}\right) .
\end{aligned}
$$

Lemma 3.5 Let $g(y, z) \in Y_{r}^{(\xi)}$. Then for $0<s \leq \pi$ and $0<l \leq \pi$,

(i) $\|\phi(\cdot, s, \cdot, l)\|_{r}=O(\xi(s, l))$;

(ii) $\| \phi(\cdot+v, \cdot+w, s, l)+\phi(\cdot+v, \cdot-w, s, l)+\phi(\cdot-v, \cdot+w, s, l)+\phi(\cdot-v, \cdot+w, s, l)+$ $\phi(\cdot-v, \cdot-w, s, l)-4 \phi(\cdot s, \cdot l) \|_{r}=\left\{\begin{array}{l}8(\xi(s, l), \\ 8(\xi(v, w))\end{array}\right.$

(iii) If $\xi(s, l)$ and $\eta(s, l)$ are as defined in Theorem 2.1, then

$$
\begin{aligned}
& \| \phi(\cdot+v, \cdot+w, s, l)+\phi(\cdot+v, \cdot-w, s, l)+\phi(\cdot-v, \cdot+w, s, l)+\phi(\cdot-v, \cdot+w, s, l)+ \\
& \phi(\cdot-v, \cdot-w, s, l)-4 \phi(\cdot s, \cdot l) \|_{r}=8\left(\eta(v, w) \frac{\xi(s, l)}{\eta(s, l)}\right), \text { where } \\
& \quad \phi(y, z ; s, l)=\frac{1}{4}[g(y+s, z+l)+g(y+s, z-l)+g(y-s, z+l) \\
& \quad+g(y-s, z-l)-4 g(y, z)] .
\end{aligned}
$$

Proof (i) Since

$$
\phi(y, z ; s, l)=\frac{1}{4}[g(y+s, z+l)+g(y+s, z-l)+g(y-s, z+l)+g(y-s, z-l)-4 g(y, z)],
$$

applying Minkowski's inequality, we have

$$
\begin{aligned}
\|\phi(\cdot, s, \cdot, l)\|_{r} \leq & \frac{1}{4} \| g(y+s, z+l)+g(y+s, z-l) \\
& +g(y-s, z+l)+g(y-s, z-l)-4 g(y, z) \|_{r} \\
= & O(\xi(s, l)) .
\end{aligned}
$$

Proof (ii) Clearly,

$$
\begin{aligned}
\mid \phi(y+ & v, z+w, s, l)+\phi(y+v, z-w, s, l)+\phi(y-v, z+w, s, l) \\
& +\phi(y-v, z-w, s, l)-4 \phi(y, z, s, l) \mid \\
\leq & \mid g(y+v+s, z+w+l)+g(y+v+s, z+w-l)+g(y+v-s, z+w+l) \\
& +g(y+v-s, z+w-l)-4 g(y+v, z+w) \mid \\
& +\mid g(y+v+s, z-w+l)+g(y+v+s, z-w-l)+g(y+v-s, z-w+l) \\
& +g(y+v-s, z-w-l)-4 g(y+v, z-w) \mid
\end{aligned}
$$




$$
\begin{aligned}
& +\mid g(y-v+s, z+w+l)+g(y-v+s, z+w-l)+g(y-v-s, z+w+l) \\
& +g(y-v-s, z+w-l)-4 g(y-v, z+w) \mid \\
& +\mid g(y-v+s, z-w+l)+g(y-v+s, z-w-l)+g(y-v-s, z-w+l) \\
& +g(y-v-s, z-w-l)-4 g(y-v, z-w) \mid \\
& -4|g(y+s, z+l)+g(y+s, z-l)+g(y-s, z+l)+g(y-s, z-l)-4 g(y, z)| .
\end{aligned}
$$

Applying Minkowski's inequality, we have

$$
\begin{aligned}
\| \phi(\cdot & +v, \cdot+w, s, l)+\phi(\cdot+v, \cdot-w, s, l)+\phi(\cdot-v, \cdot+, s, l) \\
& +\phi(\cdot-v, \cdot-w, s, l)-4 \phi(\cdot, s, \cdot, l) \|_{r} \\
\leq & \| g(\cdot+v+s, \cdot+w+l)+g(\cdot+v+s, \cdot+w-l)+g(\cdot+v-s, \cdot+w+l) \\
& +g(\cdot+v-s, \cdot+w-l)-4 g(\cdot+v, \cdot+w) \|_{r} \\
& +\| g(\cdot+v+s, \cdot-w+l)+g(\cdot+v+s, \cdot-w-l)+g(\cdot+v-s, \cdot-w+l) \\
& +f(\cdot+v-s, \cdot-w-l)-4 g(\cdot+v, \cdot-w) \|_{r} \\
& +\| g(\cdot-v+s, \cdot+w+l)+g(\cdot-v+s, \cdot+w-l)+g(\cdot-v-s, \cdot+w+l) \\
& +g(\cdot-v-s, \cdot+w-l)-4 g(\cdot-v, \cdot+w) \|_{r} \\
& +\| g(\cdot-v+s, \cdot-w+l)+g(\cdot-v+s, \cdot-w-l)+g(\cdot-v-s, \cdot-w+l) \\
& +g(\cdot-v-s, \cdot-w-l)-4 g(\cdot-v, \cdot-w) \|_{r} \\
& -4\|g(\cdot+s, \cdot+l)+g(\cdot+s, \cdot-l)+g(\cdot-s, \cdot+l)+g(\cdot-s, \cdot-l)-4 g(\cdot, \cdot)\|_{r} \\
= & 8(\xi(s, l)) .
\end{aligned}
$$

Also,

$$
\begin{aligned}
\| \phi(\cdot & +v, \cdot+w, s, l)+\phi(\cdot+v, \cdot-w, s, l)+\phi(\cdot-v, \cdot+w, s, l) \\
& +\phi(\cdot-v, \cdot-w, s, l)-4 \phi(\cdot+s, \cdot+l) \|_{r} \\
\leq & \| g(\cdot+s+v, \cdot+l+w)+g(\cdot+s+v, \cdot+l-w)+g(\cdot+s-v, \cdot+l+w) \\
& +g(\cdot+s-v, \cdot+l-w)-4 g(\cdot+s, \cdot+l) \|_{r} \\
& +\| g(\cdot+s+v, \cdot-l+w)+g(\cdot+s+v, \cdot-l-w)+g(\cdot+s-v, \cdot-l+w) \\
& +g(\cdot+s-v, \cdot-l-w)-4 g(\cdot+s, \cdot-l) \|_{r} \\
& +\| g(\cdot-s+v, \cdot+l+w)+g(\cdot-s+v, \cdot+l-w)+g(\cdot-s-v, \cdot+l+w) \\
& +g(\cdot-s-v, \cdot+l-w)-4 g(\cdot-s, \cdot+l) \|_{r} \\
& +\| g(\cdot-s+v, \cdot-l+w)+g(\cdot-s+v, \cdot-l-w)+g(\cdot-s-v, \cdot-l+w) \\
& +g(\cdot-s-v, \cdot-l-w)-4 g(\cdot-s, \cdot-l) \|_{r} \\
& -4\|g(\cdot+v, \cdot+w)+g(\cdot+v, \cdot-w)+g(\cdot-v, \cdot+w)+g(\cdot-v, \cdot-w)-4 g(\cdot, \cdot)\|_{r} \\
= & 8(\xi(v, w)) \cdot
\end{aligned}
$$


Proof (iii) Since $\eta$ is positive and nondecreasing, $s \leq v, l \leq w$, using Lemma 3.5(ii), we obtain

$$
\begin{aligned}
\| \phi(\cdot & +v, \cdot+w, s, l)+\phi(\cdot+v, \cdot-w, s, l)+\phi(\cdot-v, \cdot+w, s, l) \\
& +\phi(\cdot-v, \cdot-w, s, l)-4 \phi(\cdot s, \cdot l) \|_{r} \\
= & O(\xi(s, l)) \\
= & 8\left(\eta(s, l)\left(\frac{\xi(s, l)}{\eta(s, l)}\right)\right) \\
= & 8\left(\eta(v, w)\left(\frac{\xi(s, l)}{\eta(s, l)}\right)\right) .
\end{aligned}
$$

Since $\frac{\xi(s, l)}{\eta(s, l)}$ is positive and nondecreasing, if $v \geq s, w \geq l$, then $\frac{\xi(s, l)}{\eta(s, l)} \geq \frac{\xi(v, w)}{\eta(v, w)}$. Then by Lemma 3.5(ii) we get that

$$
\begin{aligned}
\| \phi( & +v, \cdot+w, s, l)+\phi(\cdot+v, \cdot-w, s, l)+\phi(\cdot-v, \cdot+w, s, l) \\
& +\phi(\cdot-v, \cdot-w, s, l)-4 \phi(\cdot s, \cdot l) \|_{r} \\
= & 8(\xi(s, l)) \\
= & 8\left(\eta(v, w) \frac{\xi(s, l)}{\eta(s, l)}\right) .
\end{aligned}
$$

\section{Proof of main theorem}

\subsection{Proof of Theorem 2.1}

$$
s_{p, q}(y, z)-g(y, z)=\frac{1}{\pi^{2}} \int_{0}^{\pi} \int_{0}^{\pi} \phi(y, z, v, w) \frac{\left[\sin \left(p+\frac{1}{2}\right) s\right]\left[\sin \left(q+\frac{1}{2}\right) l\right]}{4 \sin \frac{s}{2} \sin \frac{l}{2}} d s d l .
$$

Taking into account (14) and that $\tau_{p, q}(y, z)$ is double Hausdorff matrix means of $s_{p, q}(y, z)$, we write

$$
\begin{aligned}
\tau_{p, q}(y, z)-g(y, z) & =\sum_{j=0}^{p} \sum_{k=0}^{q} h_{p, q, j, k}\left\{s_{j, k}(y, z)-g(y, z)\right\} \\
& =\frac{1}{\pi^{2}} \int_{0}^{\pi} \int_{0}^{\pi} \phi(y, z, v, w) \sum_{j=0}^{p} \sum_{k=0}^{q} h_{p, q, j, k} \frac{\left[\sin \left(j+\frac{1}{2}\right) s\right]\left[\sin \left(k+\frac{1}{2}\right) l\right]}{4 \sin \frac{s}{2} \sin \frac{l}{2}} d s d l \\
& =\int_{0}^{\pi} \int_{0}^{\pi} \phi(y, z, s, l) K_{p q}(s, l) d s d l .
\end{aligned}
$$

Let

$$
\begin{aligned}
l_{p, q}(y, z) & =t_{p, q}^{\Delta_{H_{p, q}}}(y, z)-g(y, z) \\
& =\int_{0}^{\pi} \int_{0}^{\pi} \phi(y, z, s, l) K_{p q}(s, l) d s d l .
\end{aligned}
$$


Then

$$
\begin{aligned}
& l_{p, q}(y+v, z+w)+l_{p, q}(y+v, z-w)+l_{p, q}(y-v, z+w)+l_{p, q}(y-v, z-w)-4 l_{p, q}(y, z) \\
& =\int_{0}^{\pi} \int_{0}^{\pi}(\phi(y+v, z+w, s, l)+\phi(y+v, z-w, s, l)+\phi(y-v, z+w, s, l) \\
& \quad+\phi(y-v, z-w, s, l)-4 \phi(y, z, s, l)) K_{p q}(s, l) d s d l .
\end{aligned}
$$

By generalized Minkowski’s inequality

$$
\begin{aligned}
&\left\|l_{p, q}(\cdot+v, \cdot+w)+l_{p, q}(\cdot+v, \cdot-w)+l_{p, q}(\cdot-v, \cdot+w)+l_{p, q}(\cdot-v, \cdot-w)-4 l_{p, q}(\cdot, \cdot)\right\|_{r} \\
& \leq \int_{0}^{\pi} \int_{0}^{\pi} \| \phi(\cdot+v, \cdot+w, s, l)+\phi(\cdot+v, \cdot-w, s, l)+\phi(\cdot-v, \cdot+w, s, l) \\
&+\phi(\cdot-v, \cdot-w, s, l)-4 \phi(\cdot, s, \cdot, l) \|_{r} K_{p q}(s, l) d s d l \\
&=\left(\int_{0}^{\frac{1}{p+1}} \int_{0}^{\frac{1}{q+1}}+\int_{0}^{\frac{1}{p+1}} \int_{\frac{1}{q+1}}^{\pi}+\int_{\frac{1}{p+1}}^{\pi} \int_{0}^{\frac{1}{q+1}}+\int_{\frac{1}{p+1}}^{\pi} \int_{\frac{1}{q+1}}^{\pi}\right) \\
& \times \| \phi(\cdot+v, \cdot+w, s, l)+\phi(\cdot+v, \cdot-w, s, l)+\phi(\cdot-v, \cdot+w, s, l) \\
&+\phi(\cdot-v, \cdot-w, s, l)-4 \phi(\cdot, s, \cdot, l) \|_{r} K_{p q}(s, l) d s d l \\
&= I_{1}+I_{2}+I_{3}+I_{4} .
\end{aligned}
$$

Using Lemma 3.1 and Lemma 3.5(iii), we obtain

$$
\begin{aligned}
I_{1}= & \int_{0}^{\frac{1}{p+1}} \int_{0}^{\frac{1}{q+1}} \| \phi(\cdot+v, \cdot+w, s, l)+\phi(\cdot+v, \cdot-w, s, l)+\phi(\cdot-v, \cdot+w, s, l) \\
& +\phi(\cdot-v, \cdot-w, s, l)+\phi(\cdot-v, \cdot-w, s, l)-4 \phi(\cdot, s, \cdot, l) \|_{r} K_{p q}(s, l) d s d l \\
= & 8\left(\int_{0}^{\frac{1}{p+1}} \int_{0}^{\frac{1}{q+1}} \eta(v, w) \frac{\xi(s, l)}{\eta(s, l)}(p+1)(q+1) d s d l\right) \\
= & 8\left((p+1)(q+1) \eta(v, w) \int_{0}^{\frac{1}{p+1}} \int_{0}^{\frac{1}{q+1}} \frac{\xi(s, l)}{\eta(s, l)} d s d l\right) \\
= & 8\left((p+1)(q+1) \eta(v, w)\left\{\int_{0}^{\frac{1}{p+1}} \frac{\xi\left(\frac{1}{p+1}, l\right)}{\eta\left(\frac{1}{q+1}, l\right)}\right\} d l \int_{0}^{\frac{1}{q+1}} d s\right) \\
= & 8\left((p+1) \eta(v, w)\left\{\int_{0}^{\frac{1}{p+1}} \frac{\xi\left(\frac{1}{p+1}, l\right)}{\eta\left(\frac{1}{p+1}, l\right)}\right\} d l\right) \\
= & 8\left((p+1) \eta(v, w) \frac{\xi\left(\frac{1}{p+1}, \frac{1}{q+1}\right)}{\eta\left(\frac{1}{p+1}, \frac{1}{q+1}\right)}\left\{\int_{0}^{\frac{1}{p+1}} d l\right\}\right), \\
I_{1}= & 8\left(\eta(v, w) \frac{\xi\left(\frac{1}{p+1}, \frac{1}{q+1}\right)}{\xi\left(\frac{1}{p+1}, \frac{1}{q+1}\right)}\right) .
\end{aligned}
$$


Using Lemma 3.3 and Lemma 3.5(iii), we obtain

$$
\begin{aligned}
I_{2}= & \int_{0}^{\frac{1}{p+1}} \int_{\frac{1}{q+1}}^{\pi} \| \phi(\cdot+v, \cdot+w, s, l)+\phi(\cdot+v, \cdot-w, s, l)+\phi(\cdot-v, \cdot+w, s, l) \\
& +\phi(\cdot-v, \cdot-w, s, l)+\phi(\cdot-v, \cdot-w, s, l)-4 \phi(\cdot, s, \cdot, l) \|_{r} K_{p q}(s, l) d s d l \\
= & 8\left(\int_{0}^{\frac{1}{p+1}} \int_{\frac{1}{q+1}}^{\pi} \eta(v, w) \frac{\xi(s, l)}{\eta(s, l)}(p+1) \frac{1}{(q+1) l^{2}} d s d l\right) \\
= & 8\left(\left(\frac{p+1}{q+1}\right) \eta(v, w) \int_{\frac{1}{q+1}}^{\pi} \frac{\xi\left(\frac{1}{p+1}, l\right)}{\eta\left(\frac{1}{p+1}, l\right)} \frac{1}{l^{2}} d l\right) .
\end{aligned}
$$

Using Lemma 3.2 and Lemma 3.5(iii), we obtain

$$
\begin{aligned}
I_{3}= & \int_{\frac{1}{p+1}}^{\pi} \int_{0}^{\frac{1}{q+1}} \| \phi(\cdot+v, \cdot+w, s, l)+\phi(\cdot+v, \cdot-w, s, l)+\phi(\cdot-v, \cdot+w, s, l) \\
& +\phi(\cdot-v, \cdot-w, s, l)+\phi(\cdot-v, \cdot-w, s, l)-4 \phi(\cdot, s, \cdot, l) \|_{r} K_{p q}(s, l) d s d l \\
= & 8\left(\int_{\frac{1}{p+1}}^{\pi} \int_{0}^{\frac{1}{q+1}} \eta(v, w) \frac{\xi(s, l)}{\eta(s, l)}(q+1) \frac{1}{(p+1) s^{2}} d s d l\right) \\
= & 8\left(\left(\frac{q+1}{p+1}\right) \eta(v, w) \int_{\frac{1}{p+1}}^{\pi} \frac{\xi\left(s, \frac{1}{q+1}\right)}{\eta\left(s, \frac{1}{q+1}\right)} \frac{1}{s^{2}} d s\right) .
\end{aligned}
$$

Using Lemma 3.4 and Lemma 3.5(iii), we obtain

$$
\begin{aligned}
I_{4}= & \int_{\frac{1}{p+1}}^{\pi} \int_{\frac{1}{q+1}}^{\pi} \| \phi(\cdot+v, \cdot+w, s, l)+\phi(\cdot+v, \cdot-w, s, l)+\phi(\cdot-v, \cdot+w, s, l) \\
& +\phi(\cdot-v, \cdot-w, s, l)+\phi(\cdot-v, \cdot-w, s, l)-4 \phi(\cdot, s, \cdot, l) \|_{r} K_{p q}(s, l) d s d l \\
= & 8\left(\int_{\frac{1}{p+1}}^{\pi} \int_{\frac{1}{q+1}}^{\pi} \eta(v, w) \frac{\xi(s, l)}{\eta(s, l)} \frac{1}{(p+1) s^{2}} \frac{1}{(q+1) l^{2}} d s d l\right) \\
= & 8\left(\frac{1}{(p+1)} \frac{1}{(q+1)} \eta(v, w) \int_{\frac{1}{p+1}}^{\pi} \int_{\frac{1}{q+1}}^{\pi} \frac{\xi(s, l)}{\eta(s, l)} \frac{1}{s^{2}} \frac{1}{l^{2}} d s d l\right) .
\end{aligned}
$$

By (15), (16), (17), (18), and (19), we have

$$
\begin{aligned}
& \left\|l_{p, q}(\cdot+v, \cdot+w)+l_{p, q}(\cdot+v, \cdot-w)+l_{p, q}(\cdot-v, \cdot+w)+l_{p, q}(\cdot-v, \cdot-w)-4 l_{p, q}(\cdot, \cdot)\right\|_{r} \\
& =8\left(\eta(v, w) \frac{\eta\left(\frac{1}{p+1}, \frac{1}{q+1}\right)}{\eta\left(\frac{1}{p+1}, \frac{1}{q+1}\right)}\right)+8\left(\left(\frac{p+1}{q+1}\right) \eta(v, w) \int_{\frac{1}{q+1}}^{\pi} \frac{\xi\left(\frac{1}{p+1}, l\right)}{\eta\left(\frac{1}{p+1}, l\right)} \frac{1}{l^{2}} d l\right) \\
& \quad+8\left(\left(\frac{q+1}{p+1}\right) \eta(v, w) \int_{\frac{1}{p+1}}^{\pi} \frac{\xi\left(s, \frac{1}{q+1}\right)}{\eta\left(s, \frac{1}{q+1}\right)} \frac{1}{s^{2}} d s\right) \\
& \quad+8\left(\frac{1}{(p+1)(q+1)} \eta(v, w) \int_{\frac{1}{p+1}}^{\pi} \int_{\frac{1}{q+1}}^{\pi} \frac{\xi(s, l)}{\eta(s, l)} \frac{1}{s^{2}} \frac{1}{l^{2}} d s d l\right) .
\end{aligned}
$$


Thus

$$
\begin{aligned}
\sup _{\substack{v \neq 0 \\
w \neq 0}} \frac{\left\|l_{p, q}(\cdot+v, \cdot+w)+l_{p, q}(\cdot+v, \cdot-w)+l_{p, q}(\cdot-v, \cdot+w)+l_{p, q}(\cdot-v, \cdot-w)-4 l_{p, q}(\cdot, \cdot)\right\|_{r}}{\eta(v, w)} \\
=8\left(\frac{\xi\left(\frac{1}{p+1}, \frac{1}{q+1}\right)}{\eta\left(\frac{1}{p+1}, \frac{1}{q+1}\right)}\right)+O\left(\left(\frac{p+1}{q+1}\right) \int_{\frac{1}{q+1}}^{\pi} \frac{\xi\left(\frac{1}{p+1}, l\right)}{\eta\left(\frac{1}{p+1}, l\right)} \frac{1}{l^{2}} d l\right) \\
+8\left(\left(\frac{q+1}{p+1}\right) \int_{\frac{1}{p+1}}^{\pi} \frac{\xi\left(s, \frac{1}{q+1}\right)}{\eta\left(s, \frac{1}{q+1}\right)} \frac{1}{s^{2}} d s\right) \\
\quad+O\left(\frac{1}{(p+1)(q+1)} \int_{\frac{1}{p+1}}^{\pi} \int_{\frac{1}{q+1}}^{\pi} \frac{\xi(s, l)}{\eta(s, l)} \frac{1}{s^{2}} \frac{1}{l^{2}} d s d l\right) .
\end{aligned}
$$

Using Lemmas 3.1-3.4 and Lemma 3.5(i), we obtain

$$
\begin{aligned}
& \left\|l_{p, q}(\cdot, \cdot)\right\|_{r} \\
& =\left\|t_{p, q}^{\Delta_{H_{p, q}}}-g\right\|_{r} \\
& \leq\left(\int_{0}^{\frac{1}{p+1}} \int_{0}^{\frac{1}{q+1}}+\int_{0}^{\frac{1}{p+1}} \int_{\frac{1}{q+1}}^{\pi}+\int_{\frac{1}{p+1}}^{\pi} \int_{0}^{\frac{1}{q+1}}+\int_{\frac{1}{p+1}}^{\pi} \int_{\frac{1}{q+1}}^{\pi}\right)\|\phi(\cdot, s, \cdot, l)\|_{r}\left|K_{p q}(s, l)\right| d s d l \\
& =\int_{0}^{\frac{1}{p+1}} \int_{0}^{\frac{1}{q+1}}\|\phi(\cdot, s \cdot, l)\|_{r}\left|K_{p q}(s, l)\right| d s d l+\int_{0}^{\frac{1}{p+1}} \int_{\frac{1}{q+1}}^{\pi}\|\phi(\cdot, s, \cdot, l)\|_{r}\left|K_{p q}(s, l)\right| d s d l \\
& +\int_{\frac{1}{p+1}}^{\pi} \int_{0}^{\frac{1}{q+1}}\|\phi(\cdot, s \cdot, l)\|_{r}\left|K_{p q}(s, l)\right| d s d l+\int_{\frac{1}{p+1}}^{\pi} \int_{\frac{1}{q+1}}^{\pi}\|\phi(\cdot, s, \cdot, l)\|_{r}\left|K_{p q}(s, l)\right| d s d l \\
& =O\left((p+1)(q+1) \int_{0}^{\frac{1}{p+1}} \int_{0}^{\frac{1}{q+1}} \xi(s, l) d s d l\right)+O\left(\left(\frac{p+1}{q+1}\right) \int_{0}^{\frac{1}{p+1}} \int_{\frac{1}{q+1}}^{\pi} \frac{\xi(s, l)}{l^{2}} d s d l\right) \\
& +O\left(\left(\frac{q+1}{p+1}\right) \int_{\frac{1}{p+1}}^{\pi} \int_{0}^{\frac{1}{q+1}} \frac{\xi(s, l)}{s^{2}} d s d l\right)+O\left(\frac{1}{(p+1)(q+1)} \int_{\frac{1}{p+1}}^{\pi} \int_{\frac{1}{q+1}}^{\pi} \frac{\xi(s, l)}{s^{2} l^{2}} d s d l\right) \\
& =O\left(\xi\left(\frac{1}{p+1}, \frac{1}{q+1}\right)\right)+O\left(\left(\frac{p+1}{q+1}\right) \int_{0}^{\frac{1}{p+1}} \int_{\frac{1}{q+1}}^{\pi} \frac{\xi(s, l)}{l^{2}} d s d l\right) \\
& +O\left(\left(\frac{q+1}{p+1}\right) \int_{\frac{1}{p+1}}^{\pi} \int_{0}^{\frac{1}{q+1}} \frac{\xi(s, l)}{s^{2}} d s d l\right)+O\left(\frac{1}{(p+1)(q+1)} \int_{\frac{1}{p+1}}^{\pi} \int_{\frac{1}{q+1}}^{\pi} \frac{\xi(s, l)}{s^{2} l^{2}} d s d l\right) \\
& =O\left(\xi\left(\frac{1}{p+1}, \frac{1}{q+1}\right)\right)+O\left(\left(\frac{p+1}{q+1}\right) \int_{\frac{1}{q+1}}^{\pi} \frac{\xi\left(\frac{1}{p+1}, t\right)}{l^{2}} d l\right) \\
& +O\left(\left(\frac{q+1}{p+1}\right) \int_{\frac{1}{p+1}}^{\pi} \frac{\xi\left(s, \frac{1}{q+1}\right)}{s^{2}} d s\right) \\
& +O\left(\frac{1}{(p+1)(q+1)} \int_{\frac{1}{p+1}}^{\pi} \int_{\frac{1}{q+1}}^{\pi} \frac{\xi(s, l)}{s^{2} l^{2}} d s d l\right) \\
& \left\|l_{p, q}(\cdot, \cdot)\right\|_{r}^{(\eta)} \\
& =\left\|l_{p, q}(\cdot, \cdot)\right\|_{r}
\end{aligned}
$$




$$
\begin{aligned}
& +\sup _{\substack{v \neq 0 \\
w \neq 0}} \frac{\left\|l_{p, q}(\cdot+v, \cdot+w)+l_{p, q}(\cdot+v, \cdot-w)+l_{p, q}(\cdot-v, \cdot+w)+l_{p, q}(\cdot-v, \cdot-w)-4 l_{p, q}(\cdot, \cdot)\right\|_{r}}{\eta(v, w)} \\
& =O\left(\xi\left(\frac{1}{p+1}, \frac{1}{q+1}\right)\right)+O\left(\left(\frac{p+1}{q+1}\right) \int_{\frac{1}{q+1}}^{\pi} \frac{\xi\left(\frac{1}{p+1}, l\right)}{l^{2}} d l\right) \\
& +O\left(\left(\frac{q+1}{p+1}\right) \int_{\frac{1}{p+1}}^{\pi} \frac{\xi\left(s, \frac{1}{q+1}\right)}{s^{2}} d s\right)+O\left(\frac{1}{(p+1)(q+1)} \int_{\frac{1}{p+1}}^{\pi} \int_{\frac{1}{q+1}}^{\pi} \frac{\xi(s, l)}{s^{2} l^{2}} d s d l\right) \\
& +8\left(\frac{\xi\left(\frac{1}{p+1}, \frac{1}{q+1}\right)}{\eta\left(\frac{1}{p+1}, \frac{1}{q+1}\right)}\right)+8\left(\left(\frac{p+1}{q+1}\right) \int_{\frac{1}{q+1}}^{\pi} \frac{\xi\left(\frac{1}{p+1}, l\right)}{\eta\left(\frac{1}{p+1}, l\right)} \frac{1}{l^{2}} d l\right) \\
& +8\left(\left(\frac{q+1}{p+1}\right) \int_{\frac{1}{p+1}}^{\pi} \frac{\xi\left(s, \frac{1}{q+1}\right)}{\eta\left(s, \frac{1}{q+1}\right)} \frac{1}{s^{2}} d s\right) \\
& +8\left(\frac{1}{(p+1)(q+1)} \int_{\frac{1}{p+1}}^{\pi} \int_{\frac{1}{q+1}}^{\pi} \frac{\xi(s, l)}{\eta(s, l)} \frac{1}{s^{2} l^{2}}\right) .
\end{aligned}
$$

Since $\xi(s, l)=\frac{\xi(s, l)}{\eta(s, l)}$ and $\eta(s, l) \leq \eta(\pi, \pi) \frac{\xi(s, l)}{\eta(s, l)}, 0<s \leq \pi, 0<l \leq \pi$, we get

$$
\begin{aligned}
\left\|l_{p, q}(\cdot, \cdot)\right\|_{r}^{(\eta)}= & O\left(\frac{\xi\left(\frac{1}{p+1}, \frac{1}{q+1}\right)}{\eta\left(\frac{1}{p+1}, \frac{1}{q+1}\right)}\right)+O\left(\left(\frac{p+1}{q+1}\right) \int_{\frac{1}{q+1}}^{\pi} \frac{\xi\left(\frac{1}{p+1}, l\right)}{\eta\left(\frac{1}{p+1}, l\right)} \frac{1}{l^{2}} d l\right) \\
& +O\left(\left(\frac{q+1}{p+1}\right) \int_{\frac{1}{p+1}}^{\pi} \frac{\xi\left(s, \frac{1}{q+1}\right)}{\eta\left(s, \frac{1}{q+1}\right)} \frac{1}{s^{2}} d s\right) \\
& +O\left(\frac{1}{(p+1)(q+1)} \int_{\frac{1}{p+1}}^{\pi} \int_{\frac{1}{q+1}}^{\pi} \frac{\xi(s, l)}{\eta(s, l)} \frac{1}{s^{2} l^{2}} d s d l\right) .
\end{aligned}
$$

Since $\xi$ and $\eta$ are the Zygmund moduli of continuity, $\frac{\xi(s, l)}{\eta(s, l)}$ is positive and nondecreasing, and therefore

$$
\begin{aligned}
\left(\frac{p+1}{q+1}\right) \int_{\frac{1}{q+1}}^{\pi} \frac{\xi\left(\frac{1}{p+1}, l\right)}{\eta\left(\frac{1}{p+1}, l\right)} \frac{1}{l^{2}} d l & \geq \frac{\xi\left(\frac{1}{p+1}, \frac{1}{q+1}\right)}{\eta\left(\frac{1}{p+1}, \frac{1}{q+1}\right)}\left(\frac{p+1}{q+1}\right) \int_{\frac{1}{q+1}}^{\pi} \frac{d l}{l^{2}} \\
& \geq \frac{\xi\left(\frac{1}{p+1}, \frac{1}{q+1}\right)}{2 \eta\left(\frac{1}{p+1}, \frac{1}{q+1}\right)}(p+1) .
\end{aligned}
$$

Then

$$
O\left(\left(\frac{p+1}{q+1}\right) \int_{\frac{1}{q+1}}^{\pi} \frac{\xi\left(\frac{1}{p+1}, l\right)}{\eta\left(\frac{1}{p+1}, l\right)} \frac{1}{l^{2}} d l\right)=O\left(\frac{\left(\frac{p+1}{2}\right) \xi\left(\frac{1}{p+1}, \frac{1}{q+1}\right)}{\eta\left(\frac{1}{p+1}, \frac{1}{q+1}\right)}\right) .
$$

Since $\xi$ and $\eta$ are the Zygmund moduli of continuity, $\frac{\xi(s, l)}{\eta(s, l)}$ is positive and nondecreasing, and therefore

$$
\begin{aligned}
\left(\frac{q+1}{p+1}\right) \int_{\frac{1}{p+1}}^{\pi} \frac{\xi\left(s, \frac{1}{q+1}\right)}{\eta\left(s, \frac{1}{q+1}\right)} \frac{1}{s^{2}} d s & \geq \frac{\xi\left(\frac{1}{p+1}, \frac{1}{q+1}\right)}{\eta\left(\frac{1}{p+1}, \frac{1}{q+1}\right)}\left(\frac{q+1}{p+1}\right) \int_{\frac{1}{p+1}}^{\pi} \frac{d s}{s^{2}} \\
& \geq \frac{\xi\left(\frac{1}{p+1}, \frac{1}{q+1}\right)}{2 \eta\left(\frac{1}{p+1}, \frac{1}{q+1}\right)}(q+1) .
\end{aligned}
$$


Then

$$
O\left(\left(\frac{q+1}{p+1}\right) \int_{\frac{1}{p+1}}^{\pi} \frac{\xi\left(s, \frac{1}{q+1}\right)}{\eta\left(s, \frac{1}{q+1}\right)} \frac{1}{s^{2}} d s\right)=O\left(\frac{\left(\frac{q+1}{2}\right) \xi\left(\frac{1}{p+1}, \frac{1}{q+1}\right)}{\eta\left(\frac{1}{p+1}, \frac{1}{q+1}\right)}\right) .
$$

Since $\xi$ and $\eta$ are the Zygmund moduli of continuity, $\frac{\xi(s, l)}{\eta(s, l)}$ is positive and nondecreasing, and therefore

$$
\begin{aligned}
\frac{1}{(p+1)(q+1)} \int_{\frac{1}{p+1}}^{\pi} \int_{\frac{1}{q+1}}^{\pi} \frac{\xi(s, l)}{\eta(s, l)} \frac{1}{s^{2} l^{2}} d s d l & \geq \frac{\xi\left(\frac{1}{p+1}, \frac{1}{q+1}\right)}{\eta\left(\frac{1}{p+1}, \frac{1}{q+1}\right)} \frac{1}{(p+1)(q+1)} \int_{\frac{1}{p+1}}^{\pi} \int_{\frac{1}{q+1}}^{\pi} \frac{d s}{s^{2}} \frac{d l}{l^{2}} \\
& \geq \frac{\xi\left(\frac{1}{p+1}, \frac{1}{q+1}\right)}{4 \eta\left(\frac{1}{p+1}, \frac{1}{q+1}\right)}
\end{aligned}
$$

Then

$$
O\left(\frac{\xi\left(\frac{1}{p+1}, \frac{1}{q+1}\right)}{\eta\left(\frac{1}{p+1}, \frac{1}{q+1}\right)}\right)=O\left(\frac{1}{(p+1)(q+1)} \int_{\frac{1}{p+1}}^{\pi} \int_{\frac{1}{q+1}}^{\pi} \frac{\xi(s, l)}{\eta(s, l)} \frac{1}{s^{2}} \frac{1}{l^{2}} d s d l\right)
$$

By equations (24), (26), (28), and (30) we get

$$
\begin{aligned}
\left\|l_{p, q}(\cdot, \cdot)\right\|_{r}^{(\eta)}= & O\left(\frac{\xi\left(\frac{1}{p+1}, \frac{1}{q+1}\right)}{\eta\left(\frac{1}{p+1}, \frac{1}{q+1}\right)}\right)+O\left(\frac{\left(\frac{p+1}{2}\right) \xi\left(\frac{1}{p+1}, \frac{1}{q+1}\right)}{\eta\left(\frac{1}{p+1}, \frac{1}{q+1}\right)}\right) \\
& +O\left(\frac{\left(\frac{q+1}{2}\right) \xi\left(\frac{1}{p+1}, \frac{1}{q+1}\right)}{\eta\left(\frac{1}{p+1}, \frac{1}{q+1}\right)}\right)+O\left(\frac{1}{(p+1)(q+1)} \int_{\frac{1}{p+1}}^{\pi} \int_{\frac{1}{q+1}}^{\pi} \frac{\xi(s, l)}{\eta(s, l)} \frac{1}{s^{2}} \frac{1}{l^{2}} d s d l\right) \\
= & O\left(1+\frac{p+1}{2}+\frac{q+1}{2}\right) \frac{\xi\left(\frac{1}{p+1}, \frac{1}{q+1}\right)}{\eta\left(\frac{1}{p+1}, \frac{1}{q+1}\right)} \\
& +O\left(\frac{1}{(p+1)(q+1)} \int_{\frac{1}{p+1}}^{\pi} \int_{\frac{1}{q+1}}^{\pi} \frac{\xi(s, l)}{\eta(s, l)} \frac{1}{s^{2}} \frac{1}{l^{2}} d s d l\right) \\
= & O\left(1+\frac{p+1}{2}+\frac{q+1}{2}\right) O\left(\frac{1}{(p+1)(q+1)} \int_{\frac{1}{p+1}}^{\pi} \int_{\frac{1}{q+1}}^{\pi} \frac{\xi(s, l)}{\eta(s, l)} \frac{1}{s^{2}} \frac{1}{l^{2}} d s d l\right) \\
& +O\left(\frac{1}{(p+1)(q+1)} \int_{\frac{1}{p+1}}^{\pi} \int_{\frac{1}{q+1}}^{\pi} \frac{\xi(s, l)}{\eta(s, l)} \frac{1}{s^{2}} \frac{1}{l^{2}} d s d l\right) \\
= & O\left(1+\frac{p+1}{2}+\frac{q+1}{2}+1\right) O\left(\frac{1}{(p+1)(q+1)} \int_{\frac{1}{p+1}}^{\pi} \int_{\frac{1}{q+1}}^{\pi} \frac{\xi(s, l)}{\eta(s, l)} \frac{1}{s^{2}} \frac{1}{l^{2}} d s d l\right) \\
= & O\left(\frac{p+q+6}{(p+1)(q+1)}\right) O\left(\int_{\frac{1}{p+1}}^{\pi} \int_{\frac{1}{q+1}}^{\pi} \frac{\xi(s, l)}{\eta(s, l)} \frac{1}{s^{2}} \frac{1}{l^{2}} d s d l\right) .
\end{aligned}
$$

\subsection{Proof of Theorem 2.2}

$$
E_{p, q}(g)=\left\|l_{p, q}(\cdot, \cdot)\right\|_{r}^{(\eta)}=O\left(\frac{p+q+6}{(p+1)(q+1)}\right) O\left(\int_{\frac{1}{p+1}}^{\pi} \int_{\frac{1}{q+1}}^{\pi} \frac{\xi(s, l)}{\eta(s, l)} \frac{1}{s^{2}} \frac{1}{l^{2}} d s d l\right) .
$$


Since $\frac{\xi(s, l)}{\ln (s, l)}$ is positive and nonincreasing, by the second mean value theorem of integral calculus we have

$$
\begin{aligned}
E_{p, q}(g) & =O\left(\frac{p+q+6}{(p+1)(q+1)}\right) O\left(\int_{\frac{1}{p+1}}^{\pi} \frac{1}{s^{2}}\left(\int_{\frac{1}{q+1}}^{\pi} \frac{\xi(s, l)}{\eta(s, l)} \frac{1}{l^{2}} d l\right) d s\right) \\
& =O\left(\frac{p+q+6}{(p+1)(q+1)}\right) O\left(\int_{\frac{1}{p+1}}^{\pi} \frac{1}{s^{2}}\left((q+1) \frac{\xi\left(s, \frac{1}{q+1}\right)}{\eta\left(s, \frac{1}{q+1}\right)} \int_{\frac{1}{q+1}}^{\pi} \frac{d l}{l}\right) d s\right) \\
& =O\left(\frac{p+q+6}{(p+1)}\right) O\left(\int_{\frac{1}{p+1}}^{\pi} \frac{1}{s^{2}}\left(\frac{\xi\left(s, \frac{1}{q+1}\right)}{\eta\left(s, \frac{1}{q+1}\right)} \log \pi(q+1)\right) d s\right) .
\end{aligned}
$$

Again, since $\frac{\xi(s, l)}{s \eta(s, l)}$ is positive and nonincreasing, by the second mean value theorem of integral calculus we have

$$
\begin{aligned}
E_{p, q}(f) & =O\left(\frac{p+q+6}{(p+1)}\right) O\left(\log \pi(q+1) \int_{\frac{1}{p+1}}^{\pi} \frac{\xi\left(s, \frac{1}{p+1}\right)}{\eta\left(s, \frac{1}{q+1}\right)} \frac{1}{s^{2}} d s\right) \\
& =O\left(\frac{p+q+6}{(p+1)}\right) O\left(\log \pi(q+1)(p+1) \frac{\xi\left(\frac{1}{p+1}, \frac{1}{q+1}\right)}{\eta\left(\frac{1}{p+1}, \frac{1}{q+1}\right)} \int_{\frac{1}{p+1}}^{\pi} \frac{d s}{s}\right) \\
& =O\left((p+q+6) \log \pi(q+1) \frac{\xi\left(\frac{1}{p+1}, \frac{1}{q+1}\right)}{\eta\left(\frac{1}{p+1}, \frac{1}{q+1}\right)} \log \pi(p+1)\right) \\
& =O\left(\frac{\xi\left(\frac{1}{p+1}, \frac{1}{n q+1}\right)}{\eta\left(\frac{1}{p+1}, \frac{1}{q+1}\right)}(p+q+6) \log \pi(p+q+2)\right) .
\end{aligned}
$$

\section{Corollaries}

Corollary 5.1 Following Remark 1.1(i), we obtain

$$
\left\|t_{p, q}^{(C, 1,1)}(y, z)-g(y, z)\right\|_{r}^{(\eta)}=O\left(\frac{p+q+6}{(p+1)(q+1)} \int_{\frac{1}{p+1}}^{\pi} \int_{\frac{1}{q+1}}^{\pi} \frac{\xi(s, l)}{\eta(s, l) s^{2} l^{2}}\right),
$$

where $\xi$ and $\eta$ denote the moduli of continuity of second order such that $\xi(s, l) / \eta(s, l)$ is positive and nondecreasing.

Corollary 5.2 Following the Remark 1.1(i), we obtain

$$
\left\|t_{p, q}^{(E, r, r)}(y, z)-g(y, z)\right\|_{r}^{(\eta)}=O\left(\frac{p+q+6}{(p+1)(q+1)} \int_{\frac{1}{p+1}}^{\pi} \int_{\frac{1}{q+1}}^{\pi} \frac{\xi(s, l)}{\eta(s, l) s^{2} l^{2}}\right)
$$

where $\xi$ and $\eta$ denote the moduli of continuity of second order such that $\xi(s, l) / \eta(s, l)$ is positive and nondecreasing.

\section{Conclusion}

In this paper, we established the error estimate of a two-variable function $g(y, z)$ in the generalized Zygmund class $Y_{r}^{(\xi)}(r \geq 1)$ using the double Hausdorff matrix means of its double Fourier series. We have proved two results on error estimates of a two-variable function of $g$ in the generalized Zygmund class. 
Acknowledgements

Not applicable.

Funding

No funding available.

Availability of data and materials

Data sharing is not applicable to this paper as no datasets were generated or analyzed during the current study.

\section{Competing interests}

The authors declare that they have no competing interests.

\section{Authors' contributions}

The authors contributed equally and significantly in writing this paper. All authors read and approved the final manuscript.

\section{Authors' information}

H.K. Nigam, hknigam@cusb.ac.in; Supriya Rani, supriya@cusb.ac.in.

\section{Author details}

'Department of Mathematics, Central University of South Bihar, Gaya, India. ²Department of Medical Research, China Medical University Hospital, China Medical University (Taiwan), Taichung, Taiwan. ${ }^{3}$ Department of Mathematics, Aligarh Muslim University, Aligarh, India. ${ }^{4}$ Department of Computer Science and Information Engineering, Asia University, Taichung, Taiwan.

\section{Publisher's Note}

Springer Nature remains neutral with regard to jurisdictional claims in published maps and institutional affiliations.

Received: 10 March 2020 Accepted: 20 May 2020 Published online: 29 June 2020

\section{References}

1. Dhakal, B.P.: Approximation of functions belonging to Lip $\alpha$ class by matrix-Cesàro summability method. Int. Math. Forum 5(35), 1729-1735 (2010)

2. Dhakal, B.P.: Approximation of functions $f$ belonging to Lip class by $(N, p, q) C_{1}$ means of its Fourier series. Int. J. Eng. Technol. 2(3), 1-15 (2013)

3. Nigam, H.K., Hadish, M.: Functional approximation in Besov space using generalized Nörlund-Hausdorff product matrix. J. Inequal. Appl. 2019, 191 (2019)

4. Kushwaha, J.K., Dhakal, B.P.: Approximation of a function belonging to Lip $(\alpha, r)$ class by $N_{p, q} . C_{1}$ summability method of its Fourier series. Nepal J. Sci. Technol. 14(2), 117-122 (2013)

5. Tiwari, K.S., Bariwal, C.S.: The degree of approximation of functions in Hölder metric by triangular matrix method of Fourier series. Int. J. Pure Appl. Math. 76(2), 227-232 (2012)

6. Mishra, L.N., Mishra, V.N., Soravane, V.: Trigonometric approximation of functions belonging to Lipschitz class by matrix $C^{1} N_{p}$ operator of conjugate series of Fourier series. Adv. Differ. Equ. 3013, 17 (2013)

7. Mittal, M.L., Rhoades, B.E., Mishra, V.N., Singh, U.: Using infinite matrices to approximate functions of class Lip $(\alpha, p)$ using trigonometric polynomials. J. Math. Anal. Appl. 326, 667-676 (2007)

8. Singh, M.V., Mittal, M.L., Rhoades, B.E.: Approximation of functions in the generalized Zygmund class using Hausdorff means. J. Inequal. Appl. 2017, 101 (2017)

9. Mittal, M.L., Singh, M.V.: Degree of approximation of signals (functions) in Besov space using linear operators. Asian-Eur. J. Math. 9, 1650009 (2016)

10. Mohanty, M., Das, G., Beuria, S.: Degree of approximation of functions by their Fourier series in the Besov space by matrix mean. Int. J. Math. Appl. 4(2-B) (2016)

11. Mohanty, M., Das, G., Ray, B.K.: Degree of approximation of functions by their Fourier series in the Besov space by generalized matrix mean. Int. J. Math. Trends Technol. 36(1) (2016)

12. Singh, M.V., Mittal, M.L.: Approximation of functions in Besov space by deferred Cesàro mean. J. Inequal. Appl. 2016 , $118(2016)$

13. Lal, S., Shireen: Best approximation of function of generalized Zygmund class matrix-Euler summability means of Fourier series. Bull. Math. Anal. Appl. 5(4), 1-13 (2013)

14. Değer, U:: On approximation by matrix means of the multiple Fourier series in the Hölder metric. Kyungpook Math. J. 56, 57-68 (2016)

15. Móricz, F., Rhoades, B.E.: Approximation by Nörlund means of double Fourier series to continuous functions in two variables. Constr. Approx. 3, 281-296 (1987)

16. Adams, C.R.: Hausdorff transformations for double sequences. Bull. Am. Math. Soc. 39, 303-312 (1933)

17. Ramanujan, M.S.: On Hausdorff transformations for double sequences. Proc. Indian Acad. Sci., Sect. A, Phys. Sci. 42, $131-135$ (1955)

18. Ustina, F.: The Hausdorff means for double sequences. Can. Math. Bull. 10, 347-352 (1967)

19. Jiang, Z.-J.: On Stević-Sharma operator from the Zygmund space to the Bloch-Orlicz space. Adv. Differ. Equ. 2015, $228(2015)$ 